\title{
Geographie und Moral: Warum wir verpflichtet sind, etwas gegen das Weltarmutsproblem zu tun
}

\author{
B. Korf \\ Geographisches Institut, Universität Zürich, Winterthurerstr. 190, 8057 Zürich, Switzerland \\ Correspondence to: B. Korf (benedikt.korf@geo.uzh.ch)
}

Bleisch, B.: Pflichten auf Distanz: Weltarmut und individuelle Ethik, Berlin, de Gruyter, 254 pp., ISBN-13: 978-3-11-022825-0, €69.95, 2010.

Kritische Geographinnen und Geographen sind oft gut im Moralisieren. Marc Redepenning hat dieses Moralisieren so beschrieben: „Man weiss schnell, wo man zu stehen hat" (Redepenning, 2007, 97). Der Feststellung globaler Ungerechtigkeiten, von Hegemonie und Imperialismus, werde die Hoffnung auf eine bessere, zukünftige Welt entgegengesetzt. Doch diese Entgegensetzung selbst basiere auf universalisierenden Semantiken, deren definitorische Ausdifferenzierung oft unterbleibe. Nick Bromley moniert deshalb zu Recht: ,critical geography has become [...] a little too easy“ (2006:88). Oder in anderen Worten: kritische Geographie nervt, wenn sie nicht kritisch genug mit sich selbst ist. Und dennoch sollte man diesen gesellschaftskritischen Impuls nicht vorschnell abtun. Paul Cloke hat uns daran erinnert, dass es recht einfach scheint, über die Geographien der Moral zu schreiben; wie viel schwieriger es aber sei, diese Geographien auch in unserem Alltagsleben zu praktizieren (2002:587).

Der himmelschreiende Skandal globaler Armut, von Hunger und Unterernährung, ist ein Zustand, der uns nicht gleichgültig lassen kann. Wie schwierig es aber ist, aus diesem Umstand ethisch begründbare Pflichten für diejenigen abzuleiten, die in den wohlhabenden Ländern der Welt leben, zeigt das kluge Buch der Zürcher Philosophin Barbara Bleisch. Bleisch geht es dabei um die Frage, inwiefern wir ethisch verpflichtet sind, den Notleidenden, die nicht in unserem unmittelbaren Umfeld leben, Hilfe zu leisten. Sie greift damit ein Thema auf, dass in der anglophonen Humangeographie unter der Begrifflichkeit caring at a distance vor einiger Zeit problematisiert wurde, ohne dass diese Diskussion zu einem befriedigenden Abschluss gekommen wäre (vgl. Corbridge, 1993; Korf, 2006; Silk, 2004). Von Philosophinnen und Philosophen, insbesondere denjenigen, die in der analytischen
Tradition ausgebildet sind, können Geographinnen und Geographen m.E. lernen, wie wichtig eine gründliche Begriffsklärung um Fragen der Verantwortung, der Pflichten und der Gerechtigkeit ist. Dann zeigt sich, wie Bleisch anschaulich vorführt, wie kompliziert die Frage nach unserer individuellen Verantwortung für die Weltarmut und den sich daraus ergebenden moralischen Pflichten auf Distanz ist. Es geht hier um diejenigen moralischen Pflichten, die wir Wohlhabenden gegenüber der grossen Zahl von Armen und Marginalisierten in der Welt haben, die von uns nicht nur sozial, sondern grossenteils eben auch räumlich getrennt sind - deshalb: Pflichten auf Distanz.

Barbara Bleisch setzt sich dabei intensiv mit zwei Denkern auseinander, die in der Humangeographie bislang entweder ignoriert oder grundsätzlich abgelehnt wurden: Thomas Pogge und Peter Singer. Beginnen wir mit letzterem: 1972 erregte Singer mit einem Aufsatz Famine, Affluence, and Morality Aufsehen, in dem er radikale ethische Forderungen an jedes Individuum ableitete: wir seien moralisch verpflichtet, etwas Schlechtes (z.B. Hunger) zu verhindern, solange wir dafür nicht etwas von vergleichbarer moralischer Bedeutung opfern müssten. Mit anderen Worten: wir seien zu umfangreichen Hilfeleistungen gegenüber den Armen der Welt verpflichtet. Singer's Utilitarismus hat dabei keinen Blick auf Geographien - Entfernungen und räumliche Distanz spielen bei ihm keine Rolle. Man könnte auch sagen: Singer betreibt eine Enträumlichung der Moral. Deshalb auch wurde er insbesondere von feministischen Geographinnen grundlegend kritisiert, steht sein Ansatz doch diametral einer ethics of care entgegen, die gerade auf Nähe und konkrete soziale Beziehungen setzt. Auch Bleisch zeigt uns auf (in Kapitel 5 ihres Buches), dass Singer's Ansatz grundlegend kritisiert werden kann - er führt zu einer moralischen Überforderung, einige seiner Analogieschlüsse, die er zur Herleitung seiner Argumentation vorbringt, sind irreführend. Der wichtigste Kritikpunkt aus meiner Sicht ist jedoch, und hier stimme ich mit Bleisch überein, dass Singer die Weltarmutsdebatte über 
den Begriff der Hilfe und nicht der Gerechtigkeit führt, und dass er den Pflichtenbegriff einseitig auf Spenden an wohltätige Organisationen begrenzt. Und dennoch bleibt die grundlegende Intuition Singers, die sich gegen die einseitige Bevorzugung derjenigen, die wir kennen und die uns nahe stehen, wendet, ein wichtiger kritischer Impuls für die Moral der Weltarmutsdebatte.

Thomas Pogge geht in seinem 2002 erschienenen Buch World Poverty and Human Rights einen ganz anderen Weg als Singer, kommt aber ebenfalls zu sehr radikalen Schlussfolgerungen. Für Pogge ist Weltarmut nicht primär eine Frage des Leids, sondern der Verantwortung bzw. der Verstrickung in Unrecht. Laut Pogge geht es beim Weltarmutsproblem nicht darum, Hilfe zu leisten, sondern Schädigungen zu unterlassen. Pogge argumentiert, dass die Reichen den Armen in der Vergangenheit und der Gegenwart fortlaufend Schaden zufügen und damit an der Produktion von Unrecht beteiligt sind. Bleisch spricht deshalb von einer „Schädigungsthese“ und unterscheidet dabei die Frage nach der Verursachung dieser Schädigung und die Frage nach der Beseitigungspflicht dieser Schädigung.

Pogge selbst ist in der Begründung seiner These nicht zimperlich: er wendet sich insbesondere gegen die These, dass Armut vor allem auf innerstaatliche Verfehlungen (z.B. durch korrupte Eliten) zurückzuführen sei. Vielmehr würden diese innerstaatlichen Verfehlungen durch ein internationales Staatensystem gefördert, die herrschenden Regimen unabhängig von ihrer demokratischen Legitimation zwei wichtige Privilegien einräume: das Rohstoffprivileg erlaube es diesen Regimen, auf dem Territorium ihres Staates vorkommende Ressourcen zu verkaufen und über diese Erlöse eigenmächtig zu verfügen. Das Kreditprivileg wiederum ermögliche es diesen Machthabern, im Nahmen ihres Landes Kredite aufzunehmen, damit aber zum Beispiel ihren militärischen Unterdrückungsapparat zu finanzieren. Nach Pogge stützen und profitieren die Bürgerinnen und Bürger der wohlhabenden Staaten diese Institutionen und sind damit in das System der Schädigung verstrickt, woraus sich eine moralische Verpflichtung ergebe, sich aktiv gegen dieses System der Schädigung zu wenden.

Bleisch zeigt uns (im vierten Kapitel) in einer sehr differenzierten Auseinandersetzung mit diesen Thesen auf, wie viele komplizierte Fragen von Verursachung, Haftung, Verantwortung und Verpflichtung sich aus Pogge's Thesen ergeben. Letztlich stellen sich hier einerseits empirische Fragen danach, was genau denn nun die Armut und Marginalisierung verursacht (interne Faktoren oder das internationale Staatensystem). Gleichzeitig lassen sich aus der Verstrickung selbst sehr unterschiedliche moralische Begründungen ableiten, was denn nun genau getan werden sollte: „Das Auslegungsproblem, mit dem wir es hier zu tun haben“, schreibt Bleisch (S. 137, Hervorhebung im Original), ,betrifft die Art der Verbindung, die zwischen Handlung und Schaden herrschen muss, damit von der Verletzung einer negativen Pflicht die Rede sein kann." Und dennoch ist Pogge's Impuls wich- tig, da er im Gegensatz zu Singer die institutionelle Frage im Weltarmutsproblem - provokant und teilweise noch zu grob zwar - aufgeworfen hat.

Barbara Bleisch selbst argumentiert für einen Pflichtenpluralismus: Bürgerpflichten bestehen darin, als Bürgerinnen und Bürger unsere Stimme zugunsten einer gerechteren Weltordnung zu erheben. Unsere Konsumentenpflichten sieht sie darin, als Käufer, Anlegerinnen und Kundinnen sorgfältige Konsumentenentscheidungen zu treffen, die Unrecht möglichst vermeiden oder wieder gutmachen. Hilfspflichten wiederum verpflichten uns, Menschen in extremer Not Unterstützung zu gewähren. Dieser Pflichtenpluralismus ergibt sich aus einer Art Pattsituation: einerseits, so zeigt Bleisch, scheint die institutionelle Perspektive die angemessene und richtige zu sein, um das Weltarmutsproblem effektiv anzugehen (nur wenn wir das globale Staatensystem reformieren, kann sich Armut flächendeckend verringern). Doch scheint das einzelne Individuum nur begrenzte Handlungsmacht zu besitzen (die Bürgerpflichten), sich für eine gerechtere politische Rahmenordnung einzusetzen, insbesondere auch, wenn andere diesen Pflichten nicht nachkommen. Und gerade deshalb ist Bleisch zuzustimmen, dass ,die Tatsache, dass andere etwas tun könnten und tun sollten, dies aber unterlassen, kein Grund [ist], den eigenen Einsatz zu begrenzen“" (S. 203).

Es ist zu hoffen, dass viele Geographinnen und Geographen Bleisch's Buch als Einladung verstehen, sich intensiver mit der analytischen Klärung von moralischen Fragen der Verantwortung, Verpflichtung und Verstrickung in globales Unrecht zu beschäftigen, bevor sie ihre „kritische“ Stimme erheben. Denn, so hat uns Bleisch anschaulich vorgeführt, „living ethically and acting politically“ wie Paul Cloke von uns einfordert, macht vor allem eine gründliche begriffliche Klärung komplizierter ethischer Fragen notwendig - jenseits unserer gewohnten universalisierenden Moralsemantiken.

\section{Literatur}

Bromley, N.: Uncritical critical geography?, Prog. Hum. Geogr., 30, 87-94, 2006.

Cloke, P.: Deliver us from evil? Prospects for living ethically and acting politically in human geography, Prog. Hum. Geogr., 26, 587-604, 2002.

Corbridge, S.: Marxisms, modernities and moralities: Development praxis and the claims of distant strangers, Environ. Plann. D, 11, 449-472, 1993.

Korf, B.: Geographien der Moral, Geogr. Z., 94, 1-14, 2006.

Pogge, T.: World Poverty and Human Rights. Cosmopolitan Responsibilities and Reforms, Polity, Cambridge, 2002.

Redepenning, M.: Die Moral der critical geopolitics, Geogr. Z., 95, 91-104, 2007.

Silk, J.: Caring at a distance. Gift theory, aid chains and social movements, Soc. Cult. Geogr., 5, 229-251, 2004.

Singer, P.: Famine, affluence, and morality, Philosophy and Public Affairs, 1, 229-243, 1972. 